\title{
Next slide please
}

66 I THOUGHr that in the eight minutes I've got I'd bring you up to date on what our group has been doing in the last year; in a sense this is a progress report and updates the paper we gave here last year; I won't go over the nomenclature again; could I have the first slide please - oh, I think you must have someone else's box-mine is the grey one with my name on the top, no, wait a minute, not my name, whose name was it now? ah yes, you've found it; there's a red spot on the top right hand side of each slide that is the side that becomes the bottom left when you project it, OK, you've got it now, let's have a look, no, that's the last slide not the first, yes, now you've got the right one but it's on its side, what about the red dot? there are two? well anyway turn it through ninety degrees, no, the other way, yes now we're there, perhaps we could have the lights off, well I'm sorry there are probably too many words on this slide, and the printing is a bit thin; can you read it at the back? you can't; well I'd better read it out; no I won't, it's all in the paper which should be published within a month or so, and anyone who wants I'll give a preprint to afterwards, anyway, for those who can read it, this slide is a block diagram of the purification process we used and before I go any further I should mention that there are a couple of misprints: on the third row, fourth box from the left, well, of course that's the second box from the right, if you can read it, it says alkaline, now that should be acidic; also you can perhaps see the word mebmrane, that should of course be membrane; now if I can have a look at the next slide -now which one is this? ah, yes it's the scatter diagram, I haven't marked the quantities but we are plotting concentration against particle size; if I remember rightly this has been normalised; perhaps I could have the lights for a moment to check in the text, yes, here we are, well it doesn't actually say-we could work it out but it's probably not worth the time, so if I could have the lights off, let's have a look at the plot; well I think you can see a sort of linear relationship- there's a fair bit of scatter, of course, but I think the data are at least suggestive; perhaps if I held up a pointer you could see the relationship more clearly-I expect there's a pointer around somewhere, no I won't need the lights, yes here it is, now you can see the trend and there's just the hint of another trend running subparallel to it through this other cluster of points, you may see that more clearly if I slide the pointer across to the other-no, I wasn't saying next slide, just that I would slide the pointer; anyway now the next slide is up let's keep it on the screen, now this is the sort of evidence on which the data in the last slide were based; this is a thin section-it could take just a bit of focusing - yes, that's better, it's difficult to get the whole slide in focus at once, now the scale is, well that bar is one micron long, hang on what am I saying? it's ten microns long-oh dear, the chairman is giving me the two minute warning, it's difficult to give you a clear picture of this work in only eight minutes, but let's plough on, what was I saying? ah yes, that bar is ten microns long, now if we turn to the next slide, please, this is the result of a chemical analysis of the dark region that is near the centre of that thin section, is it possible to go back a slide? well not to worry, you can see in the analysis how dominant-sorry what was that? oh yes, the errors are plus or minus a per cent or sothat's the standard deviation, no it can't be, it must be the standard error of the mean-oh dear, the chairman says my time is up, can I beg half a minute-are there any more slides? really? well let's skip the next two, now this one is pretty important, it brings together several of the threads that you've probably been able to discern running through this talk, but rather than go through it in detail perhaps I should have the lights and just put up one or two key numbers on the blackboard - the chairman says there's no chalk, well it's all in the paper I was mentioning anyway perhaps I've been able to give you the gist of what we've been 99 doing, I guess that's all I've got time for. 